\title{
Efecto de la poda sobre la producción y calidad de frutos de Vitis vinifera L. Var. Sauvignon blanc en Sutamarchán - Boyacá
}

\author{
The effect of pruning on production and fruit quality of Vitis vinifera $\mathrm{L}$. \\ Var. Cabernet Sauvignon in Sutamarchán-Boyacá
}

\author{
Efeito da poda sobre produção e qualidade das frutas de Vitis vinifera L. Var \\ . Sauvignon blanc em Sutamarchán - Boyaca
}

Ingrid Y. Walteros ${ }^{1}$; Deisy C. Molano ${ }^{1}$; Pedro J. Almanza-Merchán ${ }^{2}$

Ingeniera Agrónoma, Facultad de Ciencias Agropecuarias, Universidad Pedagógica y Tecnológica de Colombia Tunja.
Ingeniero Agrónomo, PhD. Facultad de Ciencias Agropecuarias, Grupo de Investigación Ecofisiología Vegetal. Universidad Pedagó-
gica y Tecnológica de Colombia Tunja. Email: ingridwal89@hotmail.com

Recibido: Mayo 11 de 2013

Aceptado: Octubre 19 de 2013

\section{Resumen}

El manejo de la producción, con ayuda de los sistemas de poda, es un factor fundamental a tener en cuenta para la obtención de cosechas de calidad para elaboración de vino. El objetivo de la investigación fue evaluar el efecto de la poda sobre la producción y la calidad de frutos de Vitis vinifera L. Var. Sauvignon Blanc en Sutamarchán - Boyacá, además de conocer el sistema de poda que genera la mejor producción y calidad organoléptica en la uva destinada a la elaboración de vino. La poda mixta presento los mejores resultados en producción alcanzando un rendimiento de 5,93 t ha-1. Mientras que la poda larga obtuvo el mayor contenido de sólidos solubles totales $\left(22,76^{\circ}\right.$ Brix $)$ y la menor acidez total con $\left(6,88 \mathrm{~g} \mathrm{~L}^{-1}\right)$, la poda larga y mixta generaron las concentraciones más adecuadas en grados probables de alcohol (13,27\% y 13,21\%), la poda mixta genero los valores más altos de Ca 39,78 $\mathrm{mg} \mathrm{L}^{-1}$, para el Mg. La mayor concentración se obtuvo con la poda corta 33,33 $\mathrm{mg} \mathrm{L}^{-1} \mathrm{y}$ el K con 94,43 $\mathrm{mg} \mathrm{L}^{-1}$, en la poda larga. Se encontró la mayor concentración de pigmentos en la poda mixta con 303,55 $\mathrm{mg} \mathrm{L}^{-1}$ en flavonoides y 338,58 $\mathrm{mg} \mathrm{L}^{-1}$ en carotenoides, y por consiguiente una mayor intensidad de color. Lo que evidenció que las podas son factores de manejo agronómico que afectan directamente la calidad de la uva para elaboración de vinos.

Palabras claves: Sauvignon Blanc, Vitivinicultura, Trópico.

\begin{abstract}
The management of production and the use of pruning systems are essential factors to consider, because it obtains quality in crops to make wine. The purpose of this research was to evaluate the effect of pruning on production and fruit quality of Vitis vinifera L. Var. Sauvignon Blanc Sutamarchán - Boyacá. Moreover, to know which systems of pruning produces the best production and organoleptic quality in grapes in produc-
\end{abstract}


tion of wine. Mixed pruning presented the best results in production reaching a yield of $5.93 \mathrm{tha}^{-1}$, while the long pruning was the highest contents of total soluble solids $\left(22.76^{\circ}\right.$ Brix) and the lowest total acidity ( $6.88 \mathrm{~g}$ $\left.\mathrm{L}^{-1}\right)$. Long and mixed pruning generated the most appropriate concentrations in alcohol degrees $(13.27 \%$ and $13.21 \%$ ). Mixed pruning produce higher values of $\mathrm{Ca} 39.78 \mathrm{mg} \mathrm{L}^{-1}$, for $\mathrm{Mg}$, the highest concentration was obtained with short pruning $33.33 \mathrm{mg} \mathrm{L}^{-1}$ and the $\mathrm{K}$ to $94.43 \mathrm{mg} \mathrm{L}^{-1}$ in the long pruning. We found the highest concentration of pigments mixed with pruning $303.55 \mathrm{mg} \mathrm{L}^{-1}$ in flavonoids and $338.58 \mathrm{mg} \mathrm{L}^{-1}$ in carotenoids and therefore greater color intensity. This showed that the systems of pruning are factors of agronomic management that affect the grapes quality to make wine.

Key words: Sauvignon Blanc, Wine, Tropic.

\section{Resumo}

O manejo daprodução, com ajuda dos sistemas de poda, é um fator fundamentala ter em conta para a obtenção de colheitas de qualidade para a elaboração dos vinhos. O objetivo da pesquisa foi avaliar o efeito da poda sobre a produção e qualidade dos frutos de Vitis vinifera L. Var. Sauvignon Blanc em Sutamarchán - Boyacá, e conhecer qual dos sistemas de poda, gera a melhor produção e qualidade organoléptica em uva destinada para vinificação. A podamista apresento os melhores resultados em produção alcançando um rendimento de 5,93 tha-1. Enquanto que a poda longa obteve o maior contéudo de sólidos solúveis totais $(22,76$ ${ }^{\circ}$ Brix) e a menor acidez total com $\left(6,88 \mathrm{~g} \mathrm{~L}^{-1}\right)$. A poda longa e mista geraron as concentrações mais adequadas, em graus de álcool provável $\left(13,27 \%\right.$ e 13,21\%), a poda mista gêro os vaores mais altos de Ca 39,78 $\mathrm{mg} \mathrm{L}^{-1}$ de $\mathrm{Mg}$, a maior concentração foi obtida com a poda curta 33,33 mg L-1 e o K com 94,43 mg L-1, na poda longa. Encontrou-se a maior concentração de pigmentos na poda mista com 303,55 mg L-1 em flavonóides e 338,58 $\mathrm{mg} \mathrm{L}^{-1} \mathrm{em}$ carotenóides e, por conseguinte, uma maior intensidade da cor. O que evidenciou que as podas são fatores de gestão agronômicos que afetam diretamente a qualidade das uvas para vinificação.

Palavras-chave: Sauvignon Blanc, da Viticultura, Trópico .

\section{Introducción}

Boyacá cuenta con dos zonas consideradas aptas para el cultivo de la vid. La zona del Valle de Sugamuxi y la del Alto Ricaurte. En estas dos regiones de clima frío tropical se produjeron $47 \mathrm{t}$ en el año 2011, de acuerdo con Agronet (2012). Esto ha representado un rendimiento de $4,1 \mathrm{t} \mathrm{ha}^{-1}$, lo que implica una participación en la producción nacional de uva de 0,19\%. En los 18 municipios productores de uva de región del Valle del Sol, la mayoría son campesinos, que anteriormente se dedicaban al cultivo de cereales menores y papa (Espinosa, 2008), mientras que en la zona del Alto Ricaurte, la producción se hace en dos fincas de los municipios de Villa de Leyva y Sutamarchán. El viñedo Marques de Villa de Leyva se encuentra en la finca vitivinícola Ain Karim, en donde se producen vinos tropicales que, debido a las características edafoclimáticas de la zona, típicas del trópico, son catalogados como Premium, por ser competitivos con los mejores vinos de América (Camacho, 2007).

En términos generales, la calidad de la uva con características vínicas está determinada por altos contenidos de sólidos solubles, baja acidez y un $\mathrm{pH}$ ligeramente ácido, factores que favorecen una actividad microbiana regulada y con ello, lograr que el proceso de fermentación sea controlada. Esta calidad viene marcada por factores intrínsecos como la variedad y el suelo, y por otros de tipo cultural que responden a las denominaciones de origen y que condicionan la producción, composición, estado sanitario e integridad de los viñedos. (Yáñez et al., 1998; Mateus et al., 2002; Gómez, 2004).

Sugiero así: Por tanto, la producción de uva de calidad es consecuencia de un manejo adecuado del cultivo, que se logra principalmente con producciones controladas, a través de podas eficientes. Ellas facilitan la eliminación de partes vivas de la planta, para modificar el crecimiento natural de la cepa, adecuándola a las necesidades que se requieren por parte del enólogo (Matocq, 2004).

Aliquó et al. (2010) mencionan que la denominación de poda corta o larga se refiere a la longitud de la vara o sarmiento encargado de portar los futuros rácimos. Por tanto, dicha longitud está relacionada directamente con el número de yemas dejadas. Es así como el podador solo deja sarmientos denominados pulgares con 3 o 4 yemas en la poda corta. En este tipo de poda, los sarmientos tienen la fun- 
ción de portar los pámpanos o cargadores que fructificarán, y la madera de poda del siguiente ciclo de cosecha. Por su parte, en la poda larga se dejan varas hasta de 12 yemas. El sistema de poda mixta es la combinación de los dos sistemas, es decir, que en la planta están presentes tanto varas como pulgares. De ahí que la poda, en términos generales, busca la regulación de la longitud del sarmiento en el cual se insertan las yemas que darán origen a los rácimos. Es así como Torres (1993) menciona que el manejo de la poda es un factor fundamental para tener en cuenta en la obtención de cosechas de calidad.

Reynier(1989), afirma que hay que tener cuidado en el momento de seleccionar el tipo de poda a llevar a cabo cuando se habla de sistemas de podas Es así como realizar podas cortas, con baja cantidad de yemas, implicaría una disminución en la producción con un consecuente aumento del diámetro de los brotes y un incremento generalizado del vigor, situación que podría acentuarse aún más con el aborto de rácimos. Eso generaría un posible desequilibrio de la planta. La producción de la cantidad y calidad de los vinos se podría ver afectada. Recíprocamente, una excesiva carga de yemas conduce a una mayor densidad de brotes, carga frutal y área foliar, aumentando la competencia entre la fruta y los brotes, lo que ocasionaría una maduración desuniforme y una baja calidad de la fruta. Además ocasionaría una lignificación insuficiente y un debilitamiento de la planta. Según Pszczólkowski y Bordeu (1984), una carga excesiva asociada a mayor vigor incrementa el ácido málico, potasio, y pH de las bayas, al igual que una disminución del color y sólidos solubles ( $\left.{ }^{\circ} \mathrm{Brix}\right)$, reduciendo significativamente la calidad del vino.

De otra parte, en las investigaciones realizadas por Callejas et al. (2013) se menciona que la producción es controlada a través de la poda, dejando solo la cantidad de yemas suficientes para proporcionar un número de rácimos que la planta pueda soportar en forma óptima hasta la vendimia. Sin embargo, en muchas ocaciones no se toma en cuenta el potencial productivo de las plantas, es decir, la capacidad de cada una de ellas para producir biomasa, esto es una determinada cantidad de uva y follaje (Lavin et al., 2003).

En las zonas vitícolas de Boyacá, en especial en la zona del alto Ricaurte, las investigaciones sobre los posibles efectos de la poda en la maduración y producción de las variedades utilizadas en la elaboración de vinos tropicales son escasas aún. Por tanto, el objetivo de la investigación fue evaluar el efecto de la poda sobre la producción y calidad de frutos de Vitis vinifera L. Var. Sauvignon Blanc.

\section{Materiales y métodos}

La investigación se realizó en la finca Ain Karim, viñedo Marqués de Villa de Leyva (Boyacá-Colombia), situado a $5^{\circ} 39^{\prime}$ de latitud norte, $73^{\circ} 35^{\prime}$ de longitud oeste respecto al meridiano de Greenwich; a una altura de $2110 \mathrm{msnm}$. La región cuenta con un micro clima con alta radiación solar, temperaturas diurnas altas y bajas temperaturas nocturnas. Durante la investigación se presentó una precipitación acumulada de $1196,1 \mathrm{~mm}$, una temperatura promedio de $16,9^{\circ} \mathrm{C}$ con una máxima absoluta de $25,1^{\circ}$ C y una mínima de $7,6^{\circ}$ C (IDEAM, 2012).

El material vegetal evaluado correspondió a la cepa Sauvignon Blanc, injertada sobre el patrón 1103 Paulsen (Vitis Rupestris x Vitis berlandeieri). Sauvignon Blanc de 8 años de edad se caracteriza por presentar plantas vigorosas con racimos medianos, compactos, cónicos y de tamaño mediano a corto. Las bayas son amarillas-doradas, elipsoidales y de pulpa blanda sin pigmentación. Las plantas están sembradas a $1,5 \mathrm{~m} \times 1,0 \mathrm{~m}$ entre surcos y plantas, respectivamente, bajo el sistema de conducción en espaldera tipo royat o cordón bilateral y cuenta con fertirriego por goteo.

Se utilizó un diseño completamente al azar con tres tratamientos correspondientes a los tipos de poda (corta (testigo), larga y mixta). Cada tratamiento contó con 4 repeticiones. Cada unidad experimental fue compuesta por dos plantas, para un total de 24 plantas. Para el caso del testigo se dejaron dos yemas en tres pitones. En la poda larga, se dejaron cinco yemas en tres pitones y en la poda mixta se combinó la poda larga y la poda corta. Las podas se realizaron 2 meses después de la vendimia.

Al momento de la vendimia se determinó el número de racimos por planta, la masa fresca y seca de frutos, y escobajos, mediante medición directa con balanza de precisión 0,01 g, Scout pro (Ohaus). La masa seca de los frutos se obtuvo después de someterlos a secado en una mufla Memmert alemana UNB 500 durante 72 horas, a una temperatura de $95^{\circ} \mathrm{C}$. Se midieron diez bayas por planta mediante un calibrador digital Mitutoyo Absolute, para determinar el diámetro ecuatorial.

Durante la vendimia se estableció la producción total por planta. Los frutos se llevaron al laboratorio 
de fisiología vegetal de la Universidad Pedagógica y Tecnológica de Colombia - Tunja, en donde se determinaron los sólidos solubles totales (SST), con un refractómetro digital HI 96802 Hanna instruments con $\pm 0,2 \%$ Brix. El pH se determinó con un potenciómetro Schott handylab 11; la acidez total titulable (ATT) se obtuvo mediante la titulación con $\mathrm{NaOH}$ $0,1 \mathrm{~N}$ hasta $\mathrm{pH}$ 8,2, expresada como ácido tartárico (AOAC, 1990). El índice de madurez técnico (IMT) se determinó mediante la relación SST/ATT; los grados probables de alcohol (GPA) se establecieron mediante la fórmula:

$$
G P A=\left(0,675 *{ }^{\circ} \text { Brix }\right)-2,0839 \text { (1) }
$$

Los componentes químicos contenidos en el fruto de la uva (carotenos y flavonoides) y el matiz e intensidad de color, se obtuvieron a través de la extracción con etanol al 98\% (Wintermans y DeMots, 1965). Se leyeron las absorbancias (Abs) a longitudes de onda de 420, 470, 520, 630, $750 \mathrm{~nm}$ en un espectrofotómetro Milton Roy Spectronic 401, USA. La estimación del contenido de pigmentos se realizó en condiciones de baja iluminación. A partir de estos datos, se calcularon las concentraciones de antocianos totales, flavonoides, matiz e intensidad de color, según las siguientes fórmulas adaptadas de Hidalgo, 2010:

Intensidad de color IC $=(A b s 420+A b s 470+A 630)(F)$

Donde F, factor de dilución.

$$
\text { Matiz } \boldsymbol{T}=\text { Abs 420/Abs } 520
$$

$$
\text { Flavonoides }\left(\boldsymbol{m} \boldsymbol{g} \boldsymbol{L}^{-1}\right) \boldsymbol{Y}=1,442 X-0,024
$$

Donde $\mathrm{Y}=$ es la absorbancia medida y $\mathrm{X}$ el contendido de flavonoides en $\mathrm{mg}$ de quercetina

Carotenoides $\left(\mathbf{m g ~ t}^{\boldsymbol{L}^{-1}}\right)=($ Abs 470-Abs 750$)$ (Volumen del extracto $\mathrm{ml}) /\left(100^{*} \mathrm{Ei} \mathrm{cm}^{1 \%}\right)$ (Volumen filtrado L).

En donde Ei $\mathrm{cm}^{1 \%}=2500\left(100 \mathrm{ml} \mathrm{g}^{-1} \mathrm{~cm}^{-1}\right)$.

Para la extracción de los contenidos de calcio, Magnesio, Potasio se realizó una maceración de frutos con cáscara y semillas. El jugo se filtró y diluyo 1:500. Las lecturas se realizaron en el equipo de absorción atómica Unicam 969 AA Spectrometer, siguiendo la norma 975.03 (AOAC, 1996).

Los datos obtenidos fueron sometidos a un análisis de varianza (Anova), se llevó a cabo la prueba de comparación múltiple de Tukey (5\%), para el análisis se utilizó el software SAS 9.2.1e.

\section{Resultados y discusión}

\section{Producción}

Todos los parámetros de producción y crecimiento de la baya presentaron diferencias estadísticas $(P \leq 0,01)$, excepto el diámetro de la baya. La poda mixta fue la encargada de generar mayores promedios en el número de racimos (26,75 racimos/planta, figura 1A), peso fresco $(1076,48 \mathrm{~g})$ y seco de racimos $(210,66 \mathrm{~g})$, peso fresco del escobajo $(97,67 \mathrm{~g})$ y de la baya $(1,14 \mathrm{~g})$ y producción $\left(7,17 \mathrm{t} \mathrm{ha}^{-1}\right)$. Por su parte, la poda larga indujo mayor peso de escobajo $(4,16 \mathrm{~g})$ y de racimo (55 g). Con la poda corta se obtuvieron los valores más bajos en la mayoría de las variables (Figuras 1 y 2).

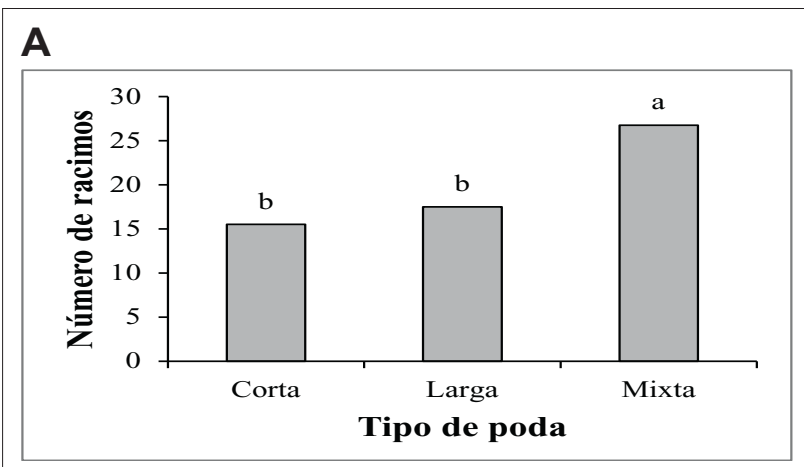

B

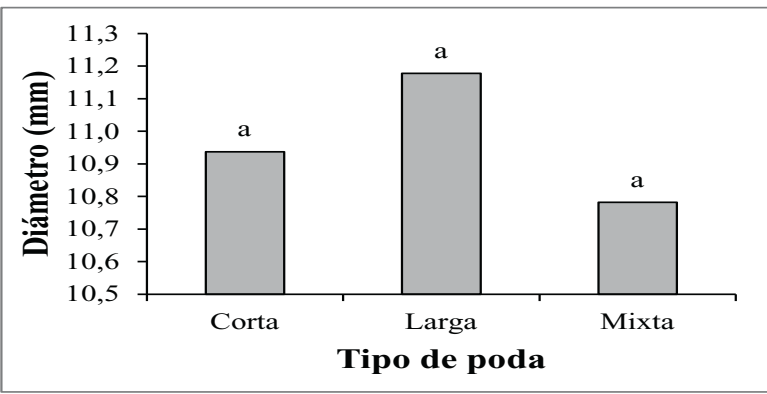

C

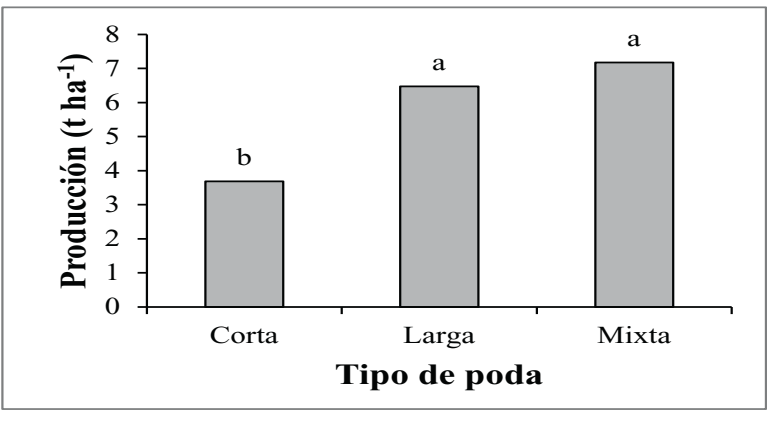

Figura 1. Efecto de diferentes tipos de poda sobre: A. Número de racimos, B. Diámetro de bayas y C. Producción de bayas de Sauvignon Blanc. Promedios seguidos de letras diferentes presentan diferencias significativas según la prueba de Tukey $(P \leq 0,05)$. 

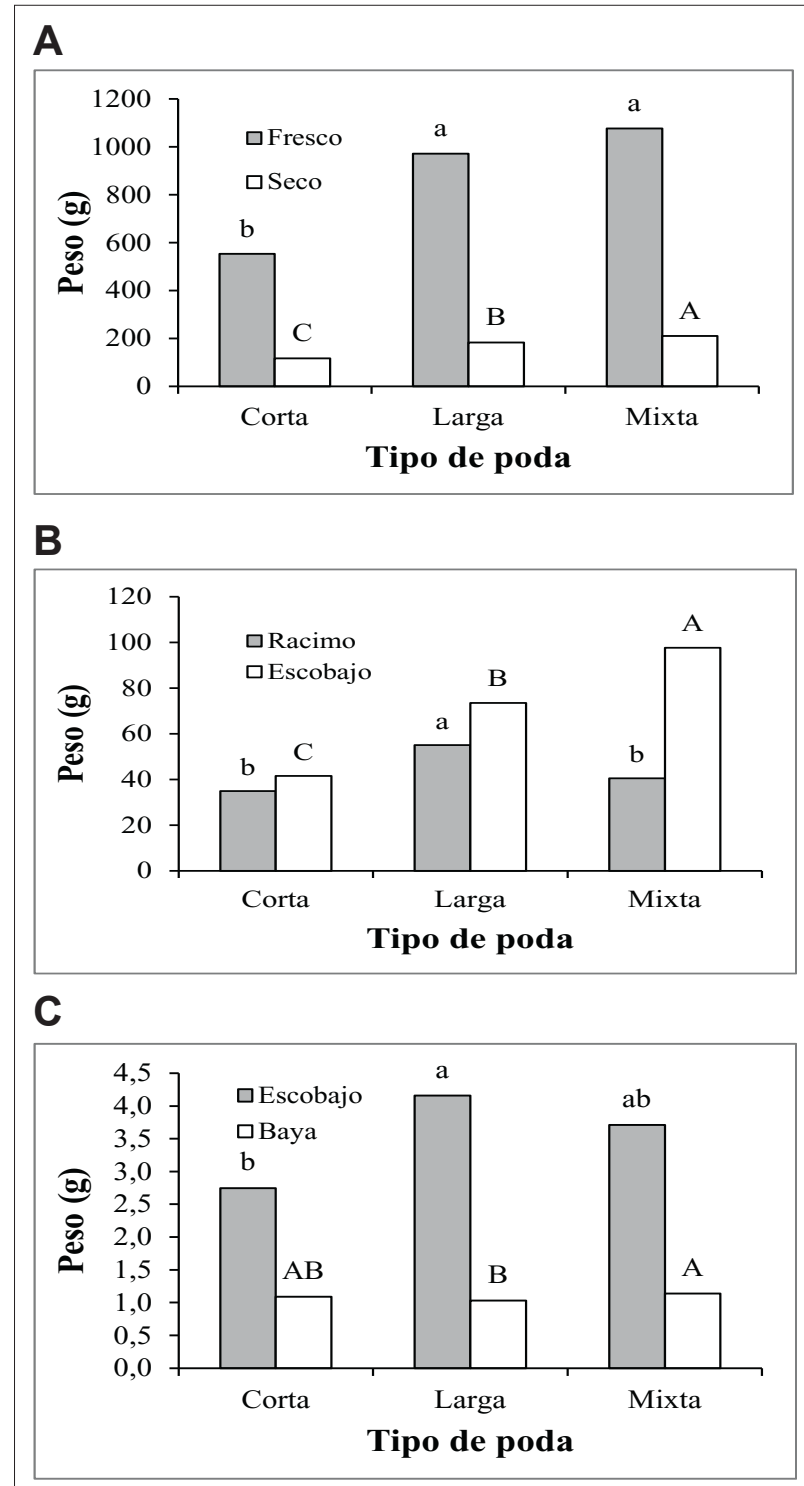

Figura 2. Efecto de diferentes tipos de poda sobre: A. Peso de racimos/planta, B. Peso fresco de 1 racimo y del escobajo y C. Peso fresco de 1 escobajo y de bayas de Sauvignon Blanc. Promedios seguidos de letras diferentes en cada serie presentan diferencias significativas según la prueba de Tukey $(P \leq 0,05)$.

En cuanto al diámetro de la baya, no se presentaron diferencias significativas. La poda larga tuvo 11,18 $\mathrm{mm}$, seguido por la poda corta con 10,94 mm y 10,78 $\mathrm{mm}$ para la mixta (figura 1B). Ruiz (1999) menciona que el tamaño del fruto es muy importante no sólo para la predicción de la producción sino también para la calidad. Es importante que el tamaño de las bayas sea relativamente pequeño para la obtención de vinos de calidad. Según Almanza (2008), los diámetros de los frutos de uva están dentro del rango de 7 a $15 \mathrm{~mm}$ para elaboración de vino en variedades como Pinot Noir y Riesling. Por tanto, los tamaños obtenidos en la investigación coinciden con estos valores.
Howell et al. (1987) afirma que las podas largas son más productivas, pues las yemas situadas entre el 3 al 10 nudo son las más fructíferas en la variedad vidal blanc. Dicho dato coincide con esta investigación, puesto que se observó que en las plantas con poda larga, los racimos se sitúan en pámpanos originados en la 3ra a 5ta yema. Sugiero: La cantidad de racimos tiene un efecto fundamental en el peso de la baya. Un incremento de carga conlleva a una reducción del peso de la baya (Petrie et al., 2004; Keller et al., 2004). Este resultado es contrario al obtenido de las podas larga y mixta que presentaron los valores más altos respecto al peso de las bayas. Sin embargo, son similares a los reportados por Amoros (2000), quien encontró que una baya de Sauvignon Blanc pesaba de 0,98 a 1,2 g y con los encontrados por Almanza (2008), quien afirma que, para elaboración de vino, los frutos están entre 1 y $2 \mathrm{~g}$, en condiciones de clima frio tropical

La producción más alta se alcanzó con la podas mixta y larga (figura 1C) respectivamente. Esto se debe a que la producción aumenta con la carga de racimos (Smart, 1990). Camacho (2007) menciona que en la uva destinada para la elaboración de vino es necesario controlar la producción para favorecer la calidad del fruto, lo que se logra obteniendo producciones de máximo $4 \mathrm{t} \mathrm{ha}^{-1}$ por ciclo de cultivo. Es posible en parte que esto se deba al hecho que la "sobrecarga" atrasa la acumulación de azúcares en la fruta cuando se compara con aquella de plantas con menos carga. Sin embargo, existen antecedentes que indican que el nivel de carga por planta no afecta la acumulación de azúcar y la calidad de la fruta (Bordelon et al., 2008).

\section{Calidad de las bayas}

El tipo de poda no afectó significativamente el $\mathrm{pH}$, los SST, la ATT y el IMT de las bayas (Figura 3). González-Padierna (2003) asegura que el pH aumenta paralelamente con el contenido en azúcares totales en baya debido a la disminución de la acidez total, y como consecuencia de la transformación de ácidos orgánicos en sales insolubles como el bitartrato potásico. La poda corta presentó los valores más altos de $\mathrm{pH}(3,12)$ seguido por la poda larga y mixta con 3,10 y 3,06 respectivamente (figura $3 \mathrm{~A}$ ). En este caso se esperaría que los valores más altos de $\mathrm{pH}$ se presentaran en la poda larga, debido a que presentaba un alto contenido de $\mathrm{K}^{+}$, de acuerdo con Boulton (1980) estos altos niveles de $\mathrm{K}^{+}$están asociados con un microclima sombreado.

La poda larga presentó frutos con 22,76 ${ }^{\circ} \mathrm{Brix}$, seguida por la poda mixta con $22,66^{\circ}$ Brix, y la poda corta con 
22,09ํㅡㄹ Brix (figura 3B). Carbonneau (2002) sostiene que el contenido de azúcares en las bayas está ligado simultáneamente a la producción total de azúcares en la cepa, y a la facilidad de migración de azúcares hacia los racimos. En este sentido, Almanza et al. (2012) mencionan que al realizar podas excesivas, se acelera el vigor de la planta y se estimula el crecimiento vegetativo. Además así se presentan abortos florales, lo que produce frutos más grandes con bajos contenidos de azúcar. En Duraznero, al realizar podas dejando mayor área foliar, se incrementó el contenido de SST debido al poder de atracción de fotosintatos ejercidos por los frutos al recibir mayor carga de asimilados (Casierra-Posada et al., 2007). Es posible que esto esté ocurriendo con los frutos de uva, que con las podas las largas tienen mayor área foliar y las podas cortas menor. Sin embargo, Bordelon et al. (2008) mencionan que el nivel de carga por planta no afecta la acumulación de azúcar ni la calidad de la fruta.

La poda larga generó el valor más bajo de ATT en los frutos con $6,88 \mathrm{~g} \mathrm{~L}^{-1} ; 7,41 \mathrm{~g} \mathrm{~L}^{-1}$ para la poda corta y $7,63 \mathrm{~g} \mathrm{~L}^{-1}$ para la poda mixta (figura 3C). Estos últimos valores difieren de los reportados por Pérez (2003), quien menciona que se deben situar entre 4,5 y 7,0 g L-1. Zamboni y Fregoni (1991) aseguran que la composición de ácidos depende de la variedad y está influenciada por factores ambientales y culturales. De acuerdo con Almanza (2011), al tener menor área foliar se presenta disminución de la acidez y ésta se debe al consumo de ácido málico durante la respiración de las células del fruto de uva. Así concuerda con Valdivia (2001), al asegurar que una menor exposición solar de los racimos en un follaje más denso, resulta en una tasa respiratoria más baja y menor consumo de ácidos orgánicos. Los ácidos orgánicos son los segundos sustratos respiratorios.

Al momento de la vendimia, la poda larga alcanzó el valor más alto en el índice de madurez técnico con 3,42, seguido por la poda corta con 3,16 y la poda mixta con 3,07 (Figura 3D); valores que se ajustan a los sugeridos para elaboración de vinos de calidad a pesar de las condiciones climáticas en la investigación. Jubileu et al. (2010) coinciden en afirmar que los SST y la ATT presentan importancia fundamental en el monitoreo del punto de cosecha de frutos, posibilitando un mejor control de la calidad de la materia prima para elaboración de vinos. Por otr lado el IMT se considera como referencia de fácil determinación desde el punto de vista vitivinícola (Blouin y Guimberteau, 2003). Por tanto, el contenido de SST y la acidez son la armonía entre estos dos gustos por ser los atributos que definen el sabor del vino blanco, una característica importante para la calidad del vino (Peynaud, 1996).

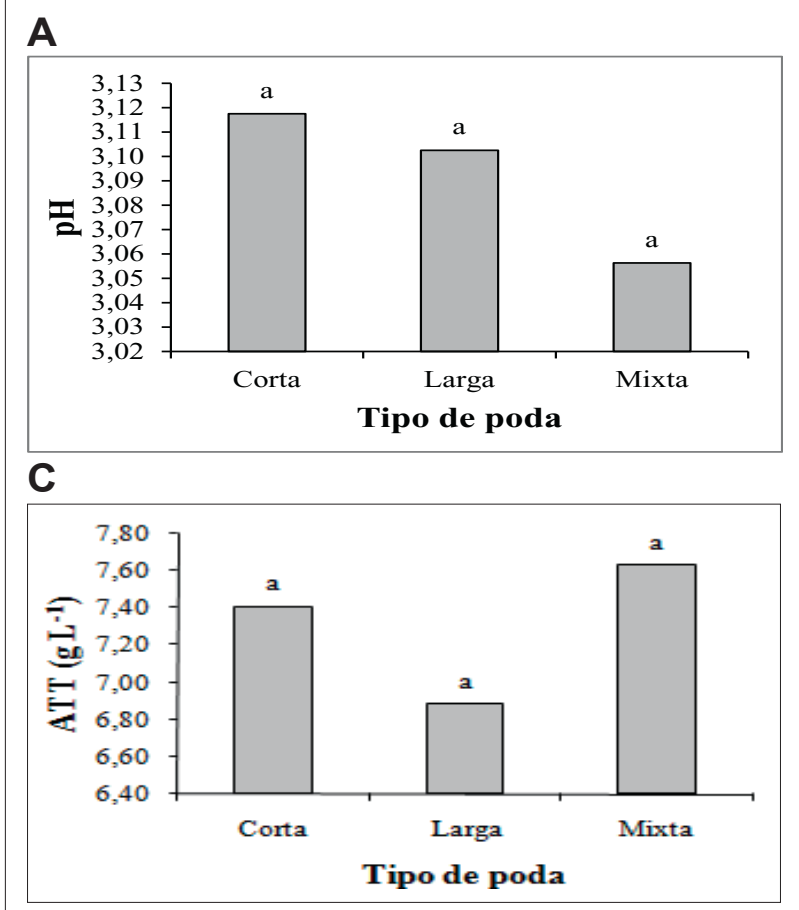

B

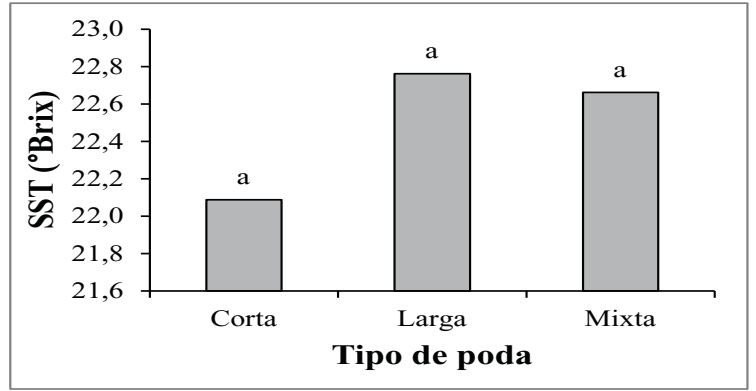

D

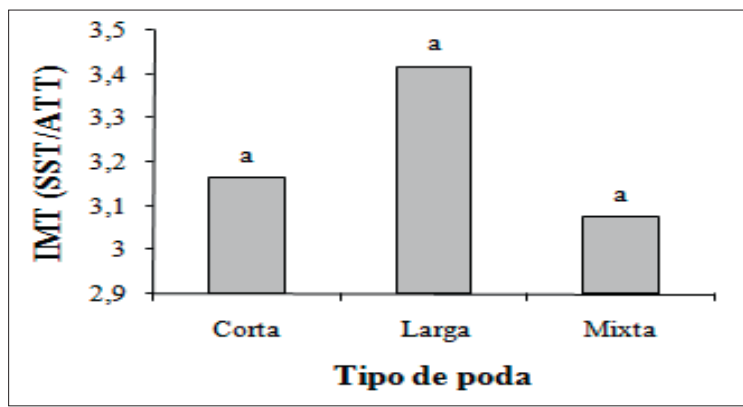

Figura 3. Efecto de diferentes tipos de poda sobre: A. el pH, B. los SST, C. la ATT y D. el IMT de bayas de Sauvignon Blanc. Promedios seguidos de letras diferentes presentan diferencias significativas según la prueba de Tukey $(P \leq 0,05)$. 


\section{Contenido Alcohólico y mineral}

El grado de alcohol probable (GAP) y la concentración de calcio no presentaron diferencias significativas. Por su parte, el Mg y el K mostraron diferencias estadísticas $(\mathrm{P} \leq 0,01)$. Para el caso del $\mathrm{Mg}$, la mayor concentración se obtuvo con la poda corta $\left(33,33 \mathrm{mg} \mathrm{L}^{-1}\right)$, mientras que con la poda larga se incrementó el contenido de

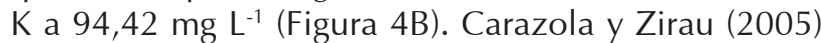
aseguran que el GPA es de gran importancia a nivel comercial, ya que los vinos y otras bebidas alcohólicas se comercializan y cotizan según su grado alcohólico. En la investigación la poda corta fue la que generó el valor más bajo (12,82\% en volumen), seguido por la poda larga y mixta con $13,27 \%$ y $13,21 \%$ de GPA, respectivamente (figura $4 \mathrm{C}$ ).

La poda mixta generó los valores más altos en contenidos de Ca con 39,78 $\mathrm{mg} \mathrm{L}^{-1}$, la poda corta con 38, $48 \mathrm{mg} \mathrm{L}^{-1} \mathrm{y}$ la poda mixta con 37, $69 \mathrm{mg} \mathrm{L}^{-1}$ (figura 4A). Estos valores difieren del valor $48,80 \mathrm{mg} \mathrm{L}^{-1}$ en vinos blancos, reportado por Fernández et al. (2009). Esto lo explica Álvarez et al. (2007) al afirmar que la concentración de minerales difieren de acuerdo a la zona geográfica de origen y está directamente relacionada con la composición del suelo. En el caso del Mg, la mayor concentración se obtuvo con la poda corta $(33,33 \mathrm{mg}$ $\mathrm{L}^{-1}$ ) (figura 4A), este valor difiere de 93,13 $\mathrm{mg} \mathrm{L}^{-1}$ en vinos blancos, reportado por Fernández et al. (2009). Las variaciones en la concentración son causadas por la composición y disponibilidad del elemento en el suelo (Nikolakaki et al., 2002).

Para Blouin y Guimberteau (2003) la presencia del Ca y $\mathrm{K}$ en los vinos, especialmente el potasio, conduce a un notable incremento del valor del $\mathrm{pH}$, lo que se traduce en una amplia relación de inconvenientes. Destacan una menor estabilidad microbiana de los vinos, una mayor facilidad en su oxidación y una dificultad para la limpieza de los mismos. También vale mencionar los problemas derivados de la insolubilización de las sales del ácido tartárico con estos cationes en forma de tartrato cálcico y bitartrato potásico, complicación que podría evidenciarse mayormente con la poda larga.

\section{Pigmentos y Color}

El tipo de poda generó diferencias significativas en los contenidos de flavonoides y carotenoides $(P \leq 0,01)$. Se encontró mayor concentración de estos compuestos con la poda mixta, con 303,55 $\mathrm{mg} \mathrm{L}^{-1}$ en flavonoides y 338,58 $\mathrm{g} \mathrm{L}^{-1}$ en carotenoides, mientras que valores más bajos furon obtenidos con la poda larga. En cuanto al color, solo hubo diferencias en el matiz. Para este caso la poda larga generó el mayor valor con 6,11 (Figura 5).

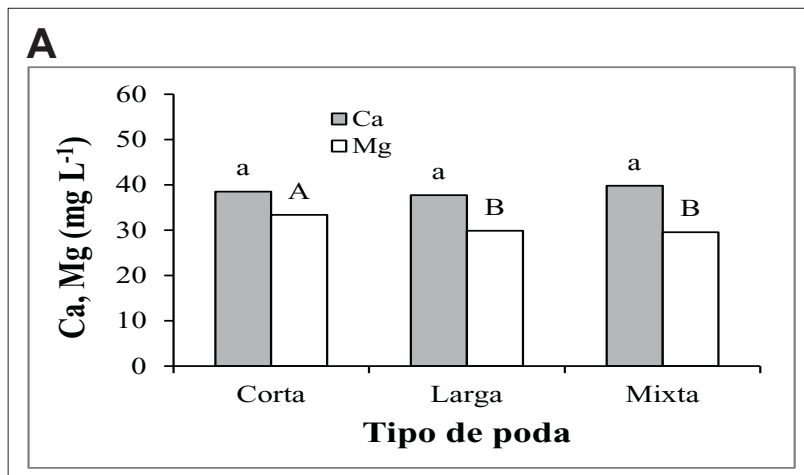

B
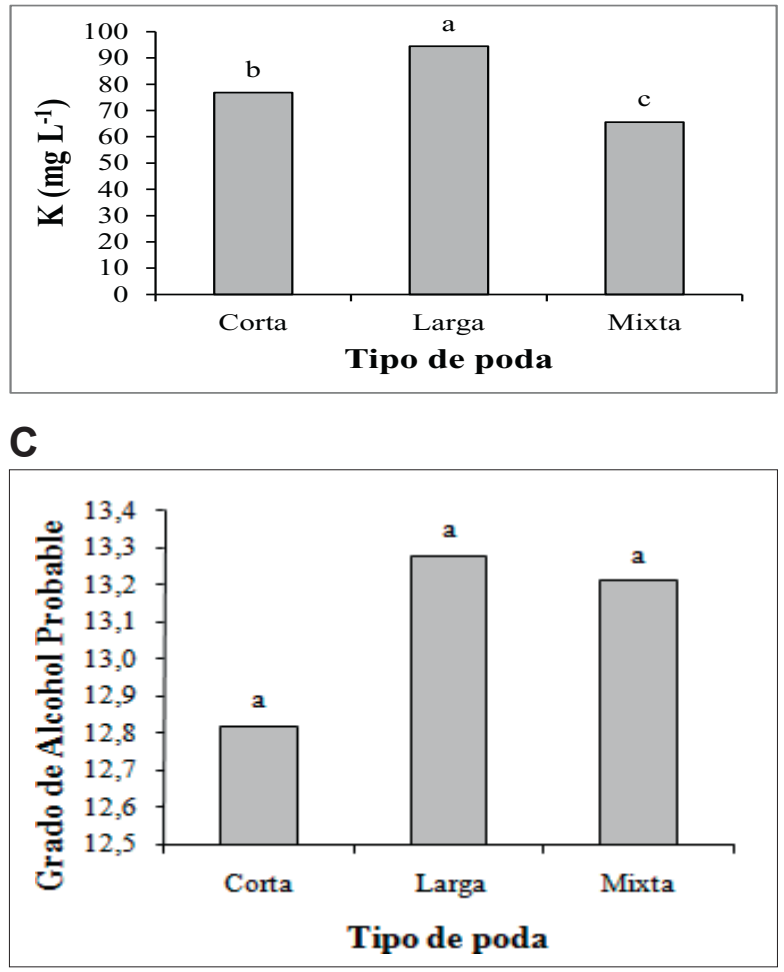

Figura 4. Efecto de diferentes tipos de poda sobre: A. Concentración de $\mathrm{Ca}$ y Mg, B. Concentración de K y C. Grado de alcohol probable de bayas de Sauvignon Blanc. Promedios seguidos de letras diferentes en cada serie presentan diferencias significativas según la prueba de Tukey $(P \leq 0,05)$.

Los flavonoles están presentes en los hollejos de las uvas blancas y tintas (pigmentos amarillos). El contenido global es casi similar entre las variedades rojas y blancas, pero su composición es muy diferente (Cheynier y Rigaud, 1986). También se encuentran en los escobajos y en las hojas como una respuesta de la planta al estímulo de la radiación UV de la luz solar (Price et al., 1995). Se ha demostrado que el contenido de quercetina es dependiente de la exposición al sol de las uvas, de la fertilización nitrogenada (KeIler y Hradzina, 1998), del estado hídrico de la planta (Ojeda, 1999) y de la cepa (Soleas et al., 1997). La 


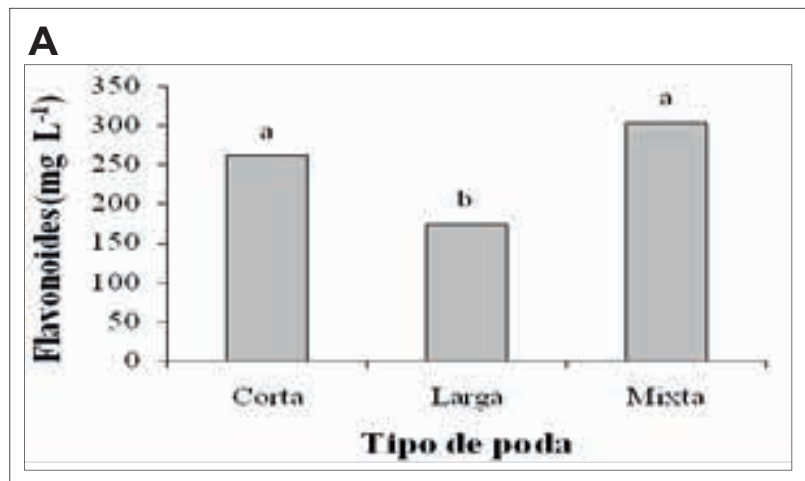

B
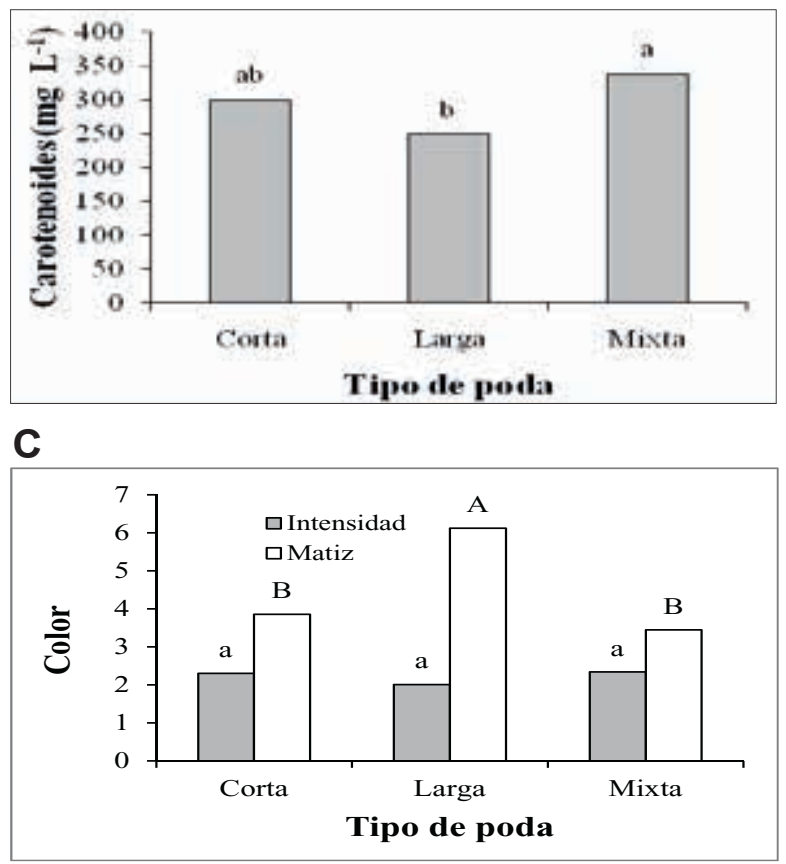

Figura 5. Efecto de diferentes tipos de poda sobre: A. Flavonoides, B. Carotenoides y C. Color de bayas de Sauvignon Blanc. Promedios seguidos de letras diferentes en cada serie presentan diferencias significativas según la prueba de Tukey $(P \leq 0,05)$.

concentración de carotenoides depende de la variedad, de la región vitícola, de la etapa de la exposición a la luz del sol y del estado de maduración del fruto (Marais et al., 2000). La pérdida de equilibrio en la relación hoja-fruto, generado por el tipo de poda, induce una menor posibilidad que las bayas logren un adecuado color de cubrimiento. La presencia de mayor fruta en la planta induce una mayor demanda de nutrientes, azúcares y agua, generando desbalances que provocan la producción de frutos con pocos contenidos de pigmentos. El ajuste del número de racimos, establecidos mediante la decisión del tipo de poda, permite equilibrar la relación fuente-vertedero, lográndose racimos con mayor coloración (Callejas 2005, Dokoozlian et al., 2000).
Un color se puede definir correctamente dentro del sistema CIELab mediante las coordenadas L*, a* y $b^{*}$, el cual define un espacio cromático en coordenadas rectangulares. Los tres ejes representan las graduaciones entre colores opuestos. Así, L* corresponde a la claridad. Fluctúa entre el blanco y el negro. $a^{*}$ y $b^{*}$ corresponden a la cromaticidad. $a^{*}$ define el componente rojo/verde y $b^{*}$ el amarillo/ azul. Estas coordenadas son una expresión numérica que representa la proporción relativa de cada uno de los colores, base necesaria para reproducir un color concreto (Zamora, 2003). C* representa la saturación o intensidad del color, es decir, la cercanía del color al gris o al matiz puro, y h define el ángulo de tono (McGuire, 1992).

Referente al color, la poda larga obtuvo un matiz de 6,11 . La mayor intensidad la presentó la poda mixta con valor de 2, 33. Es posible que a que es la que presentó los valores más altos en pigmentos (flavonoides y carotenoides), lo que implica que tienen la capacidad para actuar como fuertes copigmentos, formando complejos con las antocianas, favoreciendo la disolución y la retención de éstas en el vino. Eso se traduce en un aumento del color (Boulton, 1999). Este resultado concuerda con la investigación de Pérez et al. (2000) quienes encontraron que la reducción del color en uva red Globe, se ve disminuida cuando la incidencia de la radiación solar es menor, lo que sucede con la poda larga al tener mayor cantidad de hojas que harían un mayor efecto de sombreamiento sobre los racimos. Por tanto, la luz posee una función importante en la formación de pigmentos, convirtiendo a la intensidad y calidad de la luz en factores determinantes de la calidad del fruto (Díaz-Montenegro, 2002). Es por eso que en cultivares con alta productividad se debe regular la poda para que el desarrollo de color no se vea afectado (Muñoz y Lobato, 2000).

\section{Conclusiones}

La poda mixta presentó el mayor número de racimos/ planta, peso fresco de racimos y por ende rendimiento con 5,93 t ha-1. La poda larga obtuvo los mayores contenidos de sólidos solubles totales con 22,76 ${ }^{\circ}$ Brix y la menor acidez total con $6,88 \mathrm{~g} \mathrm{~L}^{-1}$. Lo anterior la convierte en una opción a tener en cuenta para la obtención de frutos de calidad, en la variedad Sauvignon Blanc, para elaboración de vinos.

La poda larga y mixta generaron las concentraciones más adecuadas de grados probables de alcohol $(13,27 \%$ y $13,21 \%$ en volumen). La poda mixta generó los valores más altos de Ca 39,78 $\mathrm{mg} \mathrm{L}^{-1}$, para el Mg. 
La mayor concentración se obtuvo con la poda corta con 33,33 $\mathrm{mg} \mathrm{L}^{-1}$ y el K 94,43 $\mathrm{mg} \mathrm{L}^{-1}$ con la poda larga.

La mayor concentración de pigmentos se encontró en la poda mixta con 303,55 $\mathrm{mg} \mathrm{g}^{-1}$ en flavonoides y $338,58 \mathrm{mg} \mathrm{Kg}^{-1}$ en carotenoides. Por consiguiente una mayor Intensidad de color. Lo anterior favorece que se obtengan frutos con buenas características para la elaboración de vinos blancos de calidad, bajo las condiciones climáticas presentadas durante la investigación.

\section{Referencias}

Agronet. 2012. Estadísticas por productos y departamentos. Uva 2012. En: http://www.agronet.gov.co/www/ htm3b/ReportesAjax/VerReporte.aspx. Consulta: octubre 2013.

Aliquó G, Catana A, Aguado G. 2010. La poda de la vid. Secretaría de Agricultura, Ganadería, Pesca y Alimentación - Instituto Nacional de Tecnología Agropecuaria (INTA). Lujan de Cuyo, Mendoza, Argentina. 34 p.

Almanza P, Serrano P, Fischer G. 2012. Manual de viticultura tropical. Universidad Pedagógica y Tecnológica de Colombia. 119p.

Almanza P. 2011. Determinación del crecimiento y desarrollo del fruto de vid (Vitis vinífera L.) bajo condiciones de clima frío tropical. Universidad Nacional de Colombia. Tesis de doctorado.145 p.

Almanza P. 2008. Evolución de parámetros fisicoquímicos durante la maduración de frutos de Vitis vinifera L. trabajo de ascenso en el escalafón. Universidad Pedagógica y Tecnológica de Colombia, Tunja. 64p.

Álvarez M, Moreno I, Camean A, González G. Study of mineral profile Montilla - Moriles «fino» wines using inductively coupled plasma atomic emission spectrometry methods. Journal of Food Composition and Analysis. 2007; 20:391-395.

Amoros Á. 2000. Adaptación de Variedades Blancas De Vid (Vitis Vinífera, L.) En Castilla-La Mancha. Tesis Doctoral.198p. En: http://oa.upm.es/623/1/02200036.pdf. Consulta: julio 2012.

AOAC. 1996. Official Methods of Analysis of AOAC International. $16^{\text {th }}$ ed., AOAC International. Gaithersburg, M.D. $156 p$.

Blouin J, Guimberteau G. 2003. Maduración y madurez de la uva. Mundi-Prensa. 151p.

Bordelon B, Skinkis A, Howard P. Impact of training system on vine perfomance and fruit composition of Traminette. Am J enol vitic. 2008; 59:39-46.

Boulton R. 1999. El fenómeno de copigmentación en los vinos tintos. Seminario Internacional hacia la Enología del Siglo XXI. FCA (UNCuyo) - EEA Mendoza INTA.

Boulton R. The general relationship between potasium, sodium and $\mathrm{pH}$ in grape juice and wine. Am J Enol Vitic. $1980 ; 31: 182-186$.
Callejas R, Salazar D, Rioseco M. Poda de vid de mesa basado en los componentes del rendimiento: Flame seedless como estudio de caso. Universidad de Chile. 2013; $2(1): 1-9$

Callejas R. 2005. Incremento del color de variedades rojas. Centro de estudios de la vid. Universidad Nacional de Chile. http://www.cevid.uchile.cl/articulos/ColordeCubrimientoVarRojas.pdf. Consulta: octubre 2012.

Camacho M. 2007. Nuestro vino, expectativas. En: Vinos de Colombia. http://www.vinosdecolombia.com. Consulta: abril 2012.

Carazola J, Xirau M. 2005. Técnicas usuales de análisis en enología. Panreac Química. Barcelona, España. 113 p.

Carbonneau A. 2002. Módulo 12. Ecofisiologia aplicada. Los componentes del rendimiento. Clases de la maestría de viticultura y enología. FCA UNCuyo. INTA, INRA.

Casierra F, Rodríguez J, Cárdenas J. La relación hoja/fruto afecta la producción, el crecimiento, y la calidad del fruto en duraznero (Prunus pérsica L. Batsch, cv Rubidoux), Rev Fac Nal Agr Medellín. 2007; 60(1):3657-3669.

Cheynier V, Rigaud J. 1986. Identification et dosage des flavonols du raisin. JIEP 86: 442-444.

Díaz D. 2002. Maduración de Frutos. AGT Editor, S. A. Fisiología de los árboles frutales. México. p. 230-262.

Dokoozlian N, Peacock B, Luvisi D, Ebisuda N. 2000. Actualización en prácticas culturales para las variedades de uva de mesa Crimson Sedles, Autum Royal y Melisa. En: Calidad y condición de llegada a los mercados extranjeros de la uva de mesa de exportación chilena. Pontificia Universidad Católica de Chile. Chile. p. 177-1992.

Espinosa N. 2008. Economía campesina, sistemas de producción y viticultura en el valle del sol (Boyacá). Cultura científica, versión electrónica. Fundación Universitaria Juan de Castellanos. http://www.revistasjdc.com. Consulta: abril 2012.

Fernández V, Berradre M, Sulbarán B, Ojeda G, Peña J. Caracterización química y contenido mineral en vinos comerciales venezolanos. Rev Fac Agron. (LUZ). 2009; 26:382-397.

Gómez FA. La zonificación, terroir y la denominación de origen en el fortalecimiento de los campesinos viticultores del Valle del Sol en Boyacá, Colombia Cultura científica. 2004; 1(2):3-11.

González C. 2003. Estudio ecofisiológico y agronómico de cuatro sistemas de conducción de la vid (Vitis vinífera L.): cubiertas vegetales simples versus divididas. Producción vegetal. Fitotecnia. E.T.S.I.A. Politécnica de Madrid. Madrid. 280p.

Hidalgo J. 2010. Tratado de enología. Segunda Edición. Editorial Mundi-Prensa. Madrid. 1823p

Howell GS, Mansfield TK, Wolpert JA. Influence of training system, pruning severity, and thinning on yield, vine size, and fruit quality of Vidal blanc grapevines. Am J Enol Vitic. 1987;38(2):105-112

IDEAM. 2012. Instituto de Hidrología, Meteorología y Estudios Ambientales. Sistema de Información Nacional Ambiental. Estación: 24015300 Villa de Leyva. 
Jubileu B, Sato J. Roberto S. Caracterizaçao fenológica e produtiva das videiras "Cabernet Sauvignon" e "Alicante" (Vitis vinífera L.) producidas fora de época, no norte do Paraná, Rev Bras Frutic. 2010; 32(2): 451-462.

Keller M, Hrazdina G. Interaction of nitrogen availability during bloom and light intensity during veraison. II. Effects on anthocyanin ans phenolic development during grape ripening. Am J Enol Vitic. 1998; 49:341-349.

Keller M, Mills L, Wample R, Spayd S. "Crop load management in Concord grapes using different pruning techniques". Am J Enol Vitic. 2004; 55:35-50.

Lavin A, Lobato A, Muñoz I, Valenzuela I. 2003. Viticultura: Poda de la vid. Instituto de Investigaciones Agropecuarias. Boletín INIA No. 99. Cauquenes, Chile. 52p.

Marais J, Van Wyk C, Rapp A. Carotenoid levels in maturing grapes as affected by climatic regions, sunlight and shade. S. Afr J Enol Vitic. 2000; 12(2):64-69.(7).

Mateus N, Machado J, De Freitas V. Development changes of anthocyanins in Vitis vinifera grapes grown in the Douro Valley and concentration in respective wines. Journal of the Science of Food and Agriculture, 2002; 82:1689-1695.

Matocq L. 2004. Evaluación de diferentes alternativas de control de rendimiento en vitis vinífera cv. Syrah. Tesis doctoral. En: http://bdigital.uncu.edu.ar/objetos_digitales/1116/gmatocq-vitisviniferasyrah.pdf.

McGuire R. 1992. Reporting of Objective Color Measurements. HortScience. 27(12):1254-1255.

Muñoz I, Lobato A. 2000. Prácticas culturales. En: Valenzuela J. ed. Uva de mesa en Chile. Gobierno de Chile. Ministerio de Agricultura. Instituto de Investigaciones Agropecuaria. Chile. p. 91-101.

Nikolakaki G, Kallitharakas N, Katsanos A. Trace element analysis of Cretan wines and wine products. Sci. Total Env. 2002; 285:155-163.

Ojeda H. 1999. Influence de la contrainte hydrique sur la croissance du péricarpe et sur l'évolution des phénols des baies de raisin (Vitis vinifera L.) cv. Syrah. Thèse de Doctorat, Ecole Nationale Supérieure Agronomique de Montpellier.

Pérez J, Peppi C, Larraín J. 2000. Influencia de la carga, fecha de cosecha, sombreamiento y aplicaciones de calcio sobre la calidad de la uva y la firmeza de las bayas del cv. Red Globe. En: Calidad y condición de Ilegada a los mercados extranjeros de la uva de mesa de exportación chilena. Pontificia Universidad Católica de Chile. Chile. p. 79-98.

Pérez C. 2003. Análisis (químico) y control (digital) en la producción del vino. En: http://redesformacion.jccm. es/aula_abierta/.../vino.pdf. Consulta: marzo 2012.
Petrie P, Cooley N, Clingeleffer P. The effect of post-veraison water deficit on yield components and maturation of irrigated Shiraz (Vitis vinifera L.) in the current and following season. Australian Journal of Grape and Wine Research. 2004; 10:203-215.

Peynaud E. 1996. Balance in wine. En: The taste of wine. Cap. 9, p 188-209. $2^{\circ}$ Ed. John Wiley \& Sons, Inc., New York, USA.

Price SF, Breen PJ, Valladao M, Watson BT. Cluster sun exposure and quercetin in Pinot noir grapes and wine. Am J Enol Vitic. 1995; 46:187-194.

Pszczólkowski T, Bordeu E. Posibles causas del deterioro de la calidad del vino en parronales y viñedos vigorosos. Rev. Frut. (Chile) 1984; 5(1):23-26.

Reynier A. 1989. Manual de viticultura. Cuarta edición. Ediciones Mundi-Prensa, Madrid, España. 382 p.

Ruiz M. 1999. Observación y estudios sobre el tamaño de la uva para la producción de vinos de calidad. La Semana Vitivinícola. 2749 (1207-1210).

Smart R. 1990. Principies of cannopy management. The Australian Grapegrower and Winemaker 14-15.

Soleas G, Dam J, Carey M, Goldberg DM. Toward the fingerprinting of wines: cultivar-related patterns of polyphenolic constituents in Ontario wines. J Agric Food Chem. 1997; 45:3871-3880.

Torres P. 1993. Etude de quelquestecniques en vue de maitriser le rendement de la vigne. Le progres agricole et viticole. 110 № 9. Pp 199.

Valdivia J. 2001. Efecto del vigor y del grado de madurez de las bayas sobre la fracción polifenólica de las semillas del cv. Cabernet Sauvignon en el valle del Maipo. Memoria para optar al título de Ingeniero Agrónomo. Escuela de Agronomía, Facultad de Ciencias Agronómicas, Mención Enología y Vitivinicultura. Universidad de Chile. 55 p

Yánez F, Duque M.C, Bravo de Mingo J. 1998. Riego localizado en el viñedo. En: Reunión Anual del Grupo de Trabajo en Experimentación en Viticultura y Enología. Ministerio de Agricultura, Pesca y Alimentación (Ed.), La Guardia, Álava, pp: 1-18.

Zamboni M, Fregoni M. 1991. La viticultura y la acidez del mosto Vite y Enolo. Profesional 14:29-37.

Zamora F. 2003. Elaboración y crianza del vino tinto: Aspectos científicos y prácticos. 53-58p. Ediciones Mundi-Prensa, Madrid, España. 224p.

Wintermans JF, De Mots A. Spectrophotometric characteristics of chlorophyll $\mathrm{a}$ and $\mathrm{b}$ and their pheophytins in ethanol. Biochem. Biophys. Acta 1965; 109:448-453. 